\begin{tabular}{|c|l|}
\hline Title & Projections of timelike surfaces in the de Sitter space \\
\hline Author(s) & Izumiya, Shyuichi; Tari, Farid \\
\hline Citation & Hokkaido University Preprint Series in Mathematics, 930, 1-19 \\
\hline Issue Date & 2008-12-1 \\
\hline DOI & 10.14943/84078 \\
\hline Doc URL & http://hdl.handle.net/2115/69738 \\
\hline Type & bulletin (article) \\
\hline File Information & pre930.pdf \\
\hline
\end{tabular}

Instructions for use 


\title{
Projections of timelike surfaces in the de Sitter space
}

\author{
Shyuichi Izumiya and Farid Tari
}

November 11, 2008

\begin{abstract}
We study in this paper projections of embedded timelike hypersurfaces $M$ in $S_{1}^{n}$ along geodesics. We deal in more details with the case of surfaces in $S_{1}^{3}$, characterise geometrically the singularities of the projections and prove duality results analogous to those of Shcherbak for central projections of surfaces in $\mathbb{R} P^{3}$.
\end{abstract}

\section{Introduction}

We study in this paper the contact of timelike hypersurfaces in the de Sitter space $S_{1}^{n}$ with geodesics. The contact is measured by the singularities of projections along the geodesics to transverse sets. There are three types of geodesics in $S_{1}^{n}$, spacelike, timelike and lightlike ([13]). In the case of spacelike and timelike geodesics we project, respectively, to orthogonal hyperbolic and elliptic de Sitter hyperquadrics. For lightlike geodesic, we project to a transverse space as the orthogonal space contains the geodesic. We give in section 3 the expressions for the families of projections along the three types of geodesics.

Given a point $p$ on a timelike hypersurface $M \subset S_{1}^{n}$, there is a well defined unit normal vector $e(p) \in S_{1}^{n}$ to $M$ at $p$; see [4] and section 2. If $M$ is orientable, then $e(p)$ is globally defined. However, it is always locally defined and our investigation here is local in nature. We have the (de Sitter) Gauss map

$$
\begin{aligned}
\mathbb{E}: M & \rightarrow S_{1}^{n} \\
p & \mapsto e(p)
\end{aligned}
$$

2000 Mathematics Subject classification 53A35, 53B30, 58K05.

Key Words and Phrases. Asymptotic directions, Gauss map, hyperbolic space, Legendrian duality, lightcone, principal directions, projections, singularities, de Sitter space, timelike surfaces. 
with the property that its differential map (the Weingarten map) $-d \mathbb{E}_{p}$ is a self-adjoint operator on $T_{p} M([4])$. As $M$ is timelike, the restriction of the pseudo-scalar product in the Minkowski space to $T_{p} M$ is also a pseudo scalar product. Therefore $-d \mathbb{E}_{p}$ does not always have real eigenvalues. When these are real, we call the associated eigenvectors the principal directions of $M$ at $p$. For timelike surfaces in $S_{1}^{3}$ there is a curve, labeled the lightlike principal locus in $[7,10]$ ( $L P L$ for short), that separates regions on $M$ where there are two distinct principal directions and regions where there are none. On the $L P L$ there is a unique double principal direction. One can also define the concept of asymptotic direction on surfaces in $M \subset S_{1}^{3}$. We say that $v \in T_{p} M$ is an asymptotic direction at $p \in M$ if $\left\langle d \mathbb{E}_{p}(v), v\right\rangle=0$, see section 2 for details.

We show in section 4 that the singularities of the projections of surfaces in $S_{1}^{3}$ along the three types of geodesics capture some aspects of the extrinsic geometry of the surface related to the Gauss map $\mathbb{E}$. Indeed, the singularity at $p \in M$ of a given projection is of type cusp or worse if and only if the tangent to the geodesic at $p$ is an asymptotic direction (Theorems 4.2 and 4.3). We characterise geometrically in section 4 all the generic singularities of the projections along geodesics. For instance the $L P L$ is picked up as the locus of points where the projections along the lightlike geodesics have singularities of type cusp. The projections also pick up special points on the $L P L$ (Theorem 4.3), namely the singularities of the configuration of the lines of principal curvature.

The first author introduced duality concepts between hypersurfaces in the pseudo spheres in the Minkowski space $[4,5]$; see section 6 for details. We use these concepts to prove in section 5 duality results between some surfaces associated to a timelike surface $M \subset S_{1}^{3}$ and special curves on the dual surface $M^{*}$ of $M$. The results are analogous to those of Shcherback in [16] for central projections of surfaces in $\mathbb{R} P^{3}$, and to those of Bruce-Romero Fuster in [2] for orthogonal projections of surfaces in the Euclidean space $\mathbb{R}^{3}$.

The work in this paper is part of a project on projections of submanifolds embedded in the pseudo-spheres in the Minkowski space $\mathbb{R}_{1}^{n}$ via singularity theory. We dealt in [8] with the contact of (hyper)surfaces with geodesics in the hyperbolic space (see also [11]) and in [9] with their contact with horocycles.

\section{Preliminaries}

We start by recalling some basic concepts in hyperbolic geometry (see for example [14] for details). The Minkowski $(n+1)$-space $\left(\mathbb{R}_{1}^{n+1},\langle\rangle,\right)$ is the $(n+1)$-dimensional vector space $\mathbb{R}^{n+1}$ endowed by the pseudo scalar product $\langle\boldsymbol{x}, \boldsymbol{y}\rangle=-x_{0} y_{0}+\sum_{i=1}^{n} x_{i} y_{i}$, for $\boldsymbol{x}=\left(x_{0}, \ldots, x_{n}\right)$ and $\boldsymbol{y}=\left(y_{0}, \ldots, y_{n}\right)$ in $\mathbb{R}_{1}^{n+1}$. We say that a vector $\boldsymbol{x}$ in $\mathbb{R}_{1}^{n+1} \backslash\{\mathbf{0}\}$ is spacelike, lightlike or timelike if $\langle\boldsymbol{x}, \boldsymbol{x}\rangle>0,=0$ or $<0$ respectively. The norm of a vector $\boldsymbol{x} \in \mathbb{R}_{1}^{n+1}$ is defined by $\|\boldsymbol{x}\|=\sqrt{|\langle\boldsymbol{x}, \boldsymbol{x}\rangle|}$. Given a vector $v \in \mathbb{R}_{1}^{n+1}$ and a real 
number $c$, the hyperplane with pseudo normal $v$ is defined by

$$
H P(v, c)=\left\{\boldsymbol{x} \in \mathbb{R}_{1}^{n+1} \mid\langle\boldsymbol{x}, v\rangle=c\right\}
$$

We say that $\operatorname{HP}(v, c)$ is a spacelike, timelike or lightlike hyperplane if $v$ is timelike, spacelike or lightlike respectively. We have the following three types of pseudo-spheres in $\mathbb{R}_{1}^{n+1}$ :

$$
\begin{array}{rrrl}
\text { Hyperbolic } \text {-space: } & H^{n}(-1) & =\left\{\boldsymbol{x} \in \mathbb{R}_{1}^{n+1} \mid\langle\boldsymbol{x}, \boldsymbol{x}\rangle=-1\right\}, \\
\text { de Sitter } n \text {-space: } & S_{1}^{n}=\left\{\boldsymbol{x} \in \mathbb{R}_{1}^{n+1} \mid\langle\boldsymbol{x}, \boldsymbol{x}\rangle=1\right\}, \\
\text { (open) lightcone: } & L C^{*} & =\left\{\boldsymbol{x} \in \mathbb{R}_{1}^{n+1} \backslash\{\mathbf{0}\} \mid\langle\boldsymbol{x}, \boldsymbol{x}\rangle=0\right\} .
\end{array}
$$

We also define the lightcone $(n-1)$-sphere

$$
S_{+}^{n-1}=\left\{\boldsymbol{x}=\left(x_{0}, \ldots, x_{n}\right) \mid\langle\boldsymbol{x}, \boldsymbol{x}\rangle=0, x_{0}=1\right\} .
$$

A hypersurface given by the intersection of $S_{1}^{n}$ with a spacelike (resp. timelike) hyperplane is called an elliptic hyperquadric (resp. hyperbolic hyperquadric).

A smooth embedded hypersurface $M$ in $S_{1}^{n}$ is said to be timelike if its tangent space $T_{p} M$ at any point $p \in M$ is a timelike vector space. Some aspect of the extrinsic geometry of timelike hypersurfaces in $S_{1}^{n}$ are studied in $[4,7,10]$.

Let $M$ be a timelike hypersurface embedded in $S_{1}^{n}$. Given a local chart $\boldsymbol{i}: U \rightarrow M$, where $U$ is an open subset of $\mathbb{R}^{n-1}$, we denote by $\boldsymbol{x}: U \rightarrow S_{1}^{n}$ such embedding, identify $\boldsymbol{x}(U)$ with $U$ through the embedding $\boldsymbol{x}$ and write $M=\boldsymbol{x}(U)$. Since $\langle\boldsymbol{x}, \boldsymbol{x}\rangle \equiv 1$, we have $\left\langle\boldsymbol{x}_{u_{i}}, \boldsymbol{x}\right\rangle \equiv 0$, for $i=1, \ldots, n-1$, where $u=\left(u_{1}, \ldots, u_{n-1}\right) \in U$. We define the spacelike unit normal vector $e(u)$ to $M$ at $\boldsymbol{x}(u)$ by

$$
e(u)=\frac{\boldsymbol{x}(u) \wedge \boldsymbol{x}_{u_{1}}(u) \wedge \ldots \wedge \boldsymbol{x}_{u_{n-1}}(u)}{\left\|\boldsymbol{x}(u) \wedge \boldsymbol{x}_{u_{1}}(u) \wedge \ldots \wedge \boldsymbol{x}_{u_{n-1}}(u)\right\|},
$$

where $\wedge$ denotes the wedge product of $n$ vectors in $\mathbb{R}_{1}^{n+1}$ (see for example [14]). The de Sitter Gauss map is defined in [4] by

$$
\begin{aligned}
\mathbb{E}: M & \rightarrow S_{1}^{n} \\
p & \mapsto e(p)
\end{aligned}
$$

At any $p \in M$ and $v \in T_{p} M$, one can show that $D_{v} \mathbb{E} \in T_{p} M$, where $D_{v}$ denotes the covariant derivative with respect to the tangent vector $v$. The linear transformation $A_{p}=-d \mathbb{E}(p)$ is called the de Sitter shape operator. Because the surface $M$ is timelike, the restriction of the pseudo scalar product in $\mathbb{R}_{1}^{n}$ to $M$ is a pseudo scalar product. Therefore the shape operator $A_{p}$ does not always have real eigenvalues. When these are real, we call them the principal curvatures of $M$ at $p$ and the corresponding eigenvectors are called the principal directions. 
We now review some concepts of the extrinsic geometry of embedded timelike surface $M$ in $S_{1}^{3}$, so $n=3$ above. We denote by $(u, v)$ the coordinates in $U \subset \mathbb{R}^{2}$. The first fundamental form of the surface $M$ at a point $p$ is the quadratic form $\mathrm{I}_{p}: T_{p} M \rightarrow \mathbb{R}$ given by $\mathrm{I}_{p}(v)=\langle v, v\rangle$. If $v=a \boldsymbol{x}_{u}+b \boldsymbol{x}_{v} \in T_{p} M$, then $\mathrm{I}_{p}(v)=E a^{2}+2 F a b+G b^{2}$, where

$$
E=\left\langle\boldsymbol{x}_{u}, \boldsymbol{x}_{u}\right\rangle, \quad F=\left\langle\boldsymbol{x}_{u}, \boldsymbol{x}_{v}\right\rangle, \quad G=\left\langle\boldsymbol{x}_{v}, \boldsymbol{x}_{v}\right\rangle .
$$

are the coefficients of the first fundamental form. Because $M$ is timelike, we have $E G-F^{2}<0$, so at any point $p \in M$ there are two lightlike directions in $T_{p} M$. These are the solutions of $I_{p}(v)=0$.

The second fundamental form of the surface $M$ at the point $p$ is the quadratic form $\mathrm{II}_{p}: T_{p} M \rightarrow \mathbb{R}$ given by $\mathrm{II}_{p}(v)=\left\langle A_{p}(v), v\right\rangle$, with $A_{p}=-d \mathbb{E}(p)$. For $v=a \boldsymbol{x}_{u}+b \boldsymbol{x}_{v} \in$ $T_{p} M$, we have $\operatorname{II}_{p}(v)=l a^{2}+2 m a b+n b^{2}$, where

$$
\begin{aligned}
l & =-\left\langle e_{u}, \boldsymbol{x}_{u}\right\rangle=\left\langle e, \boldsymbol{x}_{u u}\right\rangle \\
m & =-\left\langle e_{u}, \boldsymbol{x}_{v}\right\rangle=\left\langle e, \boldsymbol{x}_{u v}\right\rangle=\left\langle e, \boldsymbol{x}_{v u}\right\rangle=-\left\langle e_{v}, \boldsymbol{x}_{u}\right\rangle \\
n & =-\left\langle e_{v}, \boldsymbol{x}_{v}\right\rangle=\left\langle e, \boldsymbol{x}_{v v}\right\rangle
\end{aligned}
$$

The shape operator $A$ determines pairs of foliations on $M$ ([10]). A line of principal curvature is a curve on the surface whose tangent at all points is a principal direction. These form a pair of foliation in some region of $M$. Their equation is given by

$$
(G m-F n) d v^{2}+(G l-E n) d v d u+(F l-E m) d u^{2}=0 .
$$

The discriminant function of this equation is

$$
\delta(u, v)=\left((G l-E n)^{2}-4(G m-F n)(F l-E m)\right)(u, v) .
$$

When $\delta(u, v)>0$, there are two distinct principal directions at $p=\boldsymbol{x}(u, v)$. These coincide at points where $\delta(u, v)=0$. There are no principal directions at points where $\delta(u, v)<0$. We labeled in $[7,10]$ the locus of points where $\delta(u, v)=0$ the Lightlike Principal Locus (LPL for short).

Proposition 2.1 ([7, 10]) (1) For a generic timelike surface $M \in S_{1}^{3}$, the LPL is a curve which is smooth except at isolated points where it has Morse singularities of type node. The singular points are where the shape operator is a multiple of the identity, and are labeled "timelike umbilic points". The LPL is also the set of points on M where the two principal directions coincide and become lightlike.

(2) The LPL divides the surfaces into two regions. In one of them there are no principal directions and in the other there are two distinct principal directions at each point. In the later case, the principal directions are orthogonal and one is spacelike while the other is timelike. 
We also have the concept of asymptotic directions. A direction $v \in T_{p} M$ is called asymptotic if $\mathrm{II}_{p}(v)=\left\langle A_{p}(v), v\right\rangle=0([10])$. An asymptotic curve is a curve on the surface whose tangent at all points is an asymptotic direction. The equation of the asymptotic curves is

$$
n d v^{2}+2 m d u d v+l d u^{2}=0 .
$$

The discriminant of equation (3) is the locus of points where $m^{2}-n l$ vanishes. This is the set of points where the Gaussian curvature $K=\operatorname{det}\left(A_{p}\right)=\left(m^{2}-n l\right) /\left(F^{2}-\right.$ $E G)=0$, and is labeled the (de Sitter) parabolic set. The parabolic set of a generic surface, when not empty, is a smooth curve. It meets the $L P L$ at isolated points and the two curves are tangential at their points of intersection ([10]). In the region $K>0$ there are two distinct asymptotic directions and there are no asymptotic directions in the region $K<0$. On the parabolic set $K=0$ there is a unique asymptotic direction. This direction is lightlike at the point of tangency of the parabolic set with the $L P L$. On one side of such points, the unique asymptotic direction is spacelike and on the others side it is timelike ([10]). On the $L P L$ one of the asymptotic directions becomes lightlike and coincides with the unique principal direction there.

The generic local topological configurations of the principal and asymptotic curves are studied in [10].

Let $\gamma: I \rightarrow M \subset S_{1}^{3}$ be a regular curve on a timelike surface $M$. We can parametrise $\gamma$ by arc-length and assume that $\gamma(s)$ is unit speed, that is, $\left\langle\gamma^{\prime}(s), \gamma^{\prime}(s)\right\rangle=$ \pm 1 . Let $t(s)=\gamma^{\prime}(s)$ and $w(s)=\gamma(s) \wedge t(s) \wedge e(s)$, where $e(s)=e(\gamma(s))$. The acceleration vector $\gamma^{\prime \prime}(s)$ is written in the frame $\{\gamma(s), t(s), e(s), w(s)\}$ in the form

$$
\gamma^{\prime \prime}(s)=\mp \gamma(s)+\kappa_{n}(s) e(s)+\kappa_{g}(s) w(s)
$$

where $\kappa_{g}(s)$ is the geodesic curvature of $\gamma$ on $M$ at $\gamma(s)$. When the curve $\gamma$ is not

parametrised by arc length, we reparamatrise by arc-length $l(s)=\int_{0}^{s}\left\|\gamma^{\prime}(t)\right\| d t$ and the formula for the curvature is

$$
\kappa_{g}(t)=\frac{1}{l^{\prime}(t)^{3}}\left\langle l^{\prime}(t) \gamma^{\prime \prime}(t)-l^{\prime \prime}(t) \gamma^{\prime}(t), w(t)\right\rangle .
$$

A unit speed curve $\gamma$ is geodesic on $M$ if and only if $\kappa_{g} \equiv 0$. A point $\gamma(s)$ is called a geodesic inflection if $\kappa_{g}(s)=0$.

Definition 2.2 The flecnodal curve of a timelike surface in $S_{1}^{3}$ is the locus of geodesic inflections of the of the asymptotic curves.

\section{The family of projections along geodesics in $S_{1}^{n}$}

We exhibit in this section the expressions for the family of projections along geodesics in $S_{1}^{n}$ for $n \geq 3$ and deal in more details with the case $n=3$ in the following section. We start with projections along timelike geodesics. 
Let $H P(v, 0) \cap S_{1}^{n}, v \in H^{n}(-1)$, be (a flat) elliptic hyperquadric. Given a point $p \in S_{1}^{n}$, there is a unique timelike geodesic in $S_{1}^{n}$ which intersects orthogonally the elliptic hyperquadric at some point $q(v, p)$. We call the point $q(v, p)$ the orthogonal projection of $p$ in the direction $v$ to the elliptic hyperquadric $H P(v, 0) \cap S_{1}^{n}$, and consider the fibre bundle

$$
\pi_{12}: \Delta_{1}=\left\{(v, q) \in H^{n}(-1) \times S_{1}^{n} \mid\langle v, q\rangle=0\right\} \rightarrow S_{1}^{n}
$$

where $\pi_{12}$ is the canonical projection (see Appendix). By varying $v$, we obtain a family of orthogonal projections along timelike geodesics to elliptic hyperquadrics parametrised by vectors in $H^{n}(-1)$.

Theorem 3.1 The family of orthogonal projections in $S_{1}^{n}$ along timelike geodesics is given by

$$
\begin{array}{ccc}
P_{T}: H^{n}(-1) \times S_{1}^{n} & \rightarrow & \Delta_{1} \\
(v, p) & \mapsto(v, q(v, p))
\end{array}
$$

where $q(v, p)$ has the following expression

$$
q(v, p)=\frac{1}{\sqrt{1+\langle v, p\rangle^{2}}}(p+\langle v, p\rangle v) .
$$

Proof Let $p \in S_{1}^{n}$ and $v \in H^{n}(-1)$. A timelike geodesic passing through $p$ is parametrised by

$$
c(t)=\cosh (t) p+\sinh (t) w
$$

for some $w \in H^{n}(-1)$ tangent to the geodesic at $c(0)=p$ and with $\langle w, p\rangle=0$. At some $t_{0}$, we have $c\left(t_{0}\right)=q(v, p)$ and $c^{\prime}\left(t_{0}\right)=\sinh \left(t_{0}\right) p+\cosh \left(t_{0}\right) w=v$. So $\left\langle c^{\prime}\left(t_{0}\right), p\right\rangle=\langle v, p\rangle$, which gives $\sinh \left(t_{0}\right)=\langle v, p\rangle$. Therefore $\cosh \left(t_{0}\right)=\sqrt{1+\langle v, p\rangle^{2}}$. From this we get

$$
w=\frac{1}{\sqrt{1+\langle v, p\rangle^{2}}}(v-\langle v, p\rangle p)
$$

Substituting in (4) for $t=t_{0}$ yields $q(v, p)=(p+\langle v, p\rangle v) / \sqrt{1+\langle v, p\rangle^{2}}$.

We consider next projections along spacelike geodesics. Let $H P(v, 0) \cap S_{1}^{n}$ be a hyperbolic hyperquadric, so $v \in S_{1}^{n}$. Given a point $p \in S_{1}^{n}-\{ \pm v\}$, there is a unique spacelike geodesic in $S_{1}^{n}$ which intersects orthogonally $H P(v, 0) \cap S_{1}^{n}$ at two points $q^{ \pm}(v, p)$. The points $p= \pm v$ are excluded as all the spacelike geodesics orthogonal to $H P(v, 0) \cap S_{1}^{n}$ pass through these two points. Therefore, their projection is not well 
defined. We call the points $q^{ \pm}(v, p)$ the orthogonal projection of $p$ in the direction $v$ to the hyperbolic hyperquadric $H P(v, 0) \cap S_{1}^{n}$. We consider the fibre bundle

$$
\pi_{52}: \Delta_{5}=\left\{(v, q) \in S_{1}^{n} \times S_{1}^{n} \mid\langle v, q\rangle=0\right\} \rightarrow S_{1}^{n}
$$

with $\pi_{52}$ the canonical projection to the second component (see Appendix). By varying $v$, we obtain a family of orthogonal projections along spacelike geodesic to hyperbolic hyperquadrics parametrised by vectors in $S_{1}^{n}$.

Theorem 3.2 The family of orthogonal projections in $S_{1}^{n}$ along spacelike geodesics is given by

$$
\begin{aligned}
& P_{S}: S_{1}^{n} \times S_{1}^{n}-\left\{(v, \pm v), v \in S_{1}^{n}\right\} \quad \rightarrow \quad \Delta_{5} \\
& (v, p) \quad \mapsto\left(v, q^{ \pm}(v, p)\right)
\end{aligned}
$$

where $q^{ \pm}(v, p)$ has the following expression

$$
q^{ \pm}(v, p)= \pm \frac{1}{\sqrt{1-\langle v, p\rangle^{2}}}(p-\langle v, p\rangle v) .
$$

Proof Let $(v, p) \in S_{1}^{n} \times S_{1}^{n}$. A spacelike geodesic passing through $p$ is parametrised by

$$
c(t)=\cos (t) p+\sin (t) w
$$

for some $w \in S_{1}^{n}$ tangent to the geodesic at $c(0)=p$ and with $\langle w, p\rangle=0$. At some $t_{0}$, we have $c\left(t_{0}\right)=q(v, p)$ and $c^{\prime}\left(t_{0}\right)=-\sin \left(t_{0}\right) p+\cos \left(t_{0}\right) w=v$. So $\left\langle c^{\prime}\left(t_{0}\right), p\right\rangle=\langle v, p\rangle$, which gives $\sin \left(t_{0}\right)=-\langle v, p\rangle$. Therefore $\cos \left(t_{0}\right)= \pm \sqrt{1-\langle v, p\rangle^{2}}$. We have $\langle v, p\rangle^{2}=1$ if and only if $p= \pm v$ and this is excluded. So,

$$
w= \pm \frac{1}{\sqrt{1-\langle v, p\rangle^{2}}}(v-\langle v, p\rangle p)
$$

Substituting in (5) for $t=t_{0}$ we get $q^{ \pm}(v, p)= \pm(p-\langle v, p\rangle v) / \sqrt{1-\langle v, p\rangle^{2}}$. The hyperbolic hyperquadric $H P(v, 0) \cap S_{1}^{n}$ has two connected components, $q^{+}(v, p)$ lies on one component and $q^{-}(v, p)$ on the other.

We consider now projections along lightlike geodesics, which are lines in $S_{1}^{n}$ parallel to lightlike vectors. An orthogonal space to a lightlike geodesic contains the geodesic, so we cannot define projections along lightlike geodesics to orthogonal spaces (which are cylinders). We shall fix instead a space transverse to all lightlike geodesics in $S_{1}^{n}$ and project to this space. We denote by $\left\{e_{0}, \ldots, e_{n}\right\}$ the canonical basis of $\mathbb{R}_{1}^{n+1}$. 
Any lightlike geodesic intersects transversally the elliptic de Sitter quadric $S^{n-1}=$ $H P\left(e_{0}, 0\right) \cap S_{1}^{n}$, so we take $S^{n-1}$ as the space to project to.

We fix a point in $S^{n-1}$, say $e_{1}=(0,1,0, \ldots, 0)$. A lightlike line through $e_{1}$ is parametrised by $e_{1}+t v, t \in \mathbb{R}$, where $v \in S_{+}^{n-1} \subset L C^{*}$. This line lies is $S_{1}^{n}$ if and only if $\left\langle v, e_{1}\right\rangle=0$. So the lightlike geodesics in $S_{1}^{n}$ that pass through $e_{1}$ can be parametrised by

$$
S_{+}^{n-2}=\left\{\left(1,0, v_{2}, \ldots, v_{n}\right) \in \mathbb{R}^{n+1}: v_{2}^{2}+\ldots+v_{n}^{2}=1\right\} \subset S_{+}^{n-1} \subset L C^{*} .
$$

Any lightlike geodesic in $S_{1}^{n}$ can be obtained, by a rotation, from a lightlike geodesic through $e_{1}$. The rotation is in the form $A=\operatorname{Id}_{e_{0}} \times B$, where $B$ is a rotation in $S^{n-1}=H P\left(e_{0}, 0\right) \cap S_{1}^{n}$. Given $v \in S_{+}^{n-2}$, the geodesics $A\left(e_{1}\right)+t A(v), t \in \mathbb{R}$, obtained by varying $B \in \mathrm{SO}(n-1)$ foliate $S_{1}^{n}$. We can now define the projection in $S_{1}^{n}$ along lightlike geodesics as follows.

Given a point $p \in S_{1}^{n}$ and $v \in S_{+}^{n-2}$, there exists a unique $A=\operatorname{Id}_{e_{0}} \times B$, with $B \in \mathrm{SO}(n-1)$, such that $A(p)$ belongs to the lightlike geodesic $e_{1}+t v$. Then $A(p)=$ $e_{1}+\left\langle e_{0}, p\right\rangle v$. We define the lightlike projection of $p$ to $S^{n-1}$ along the direction $v$ as the point

$$
q(v, p)=A^{-1}\left(e_{1}\right)=A^{-1}\left(A(p)-\left\langle e_{0}, p\right\rangle v\right)=p-\left\langle e_{0}, p\right\rangle A^{-1}(v) .
$$

Definition 3.3 The family of projections along lightlike geodesics to the de Sitter elliptic hyperquadric $S^{n-1}$ is defined by

$$
\begin{aligned}
& P_{L}: S_{+}^{n-2} \times S_{1}^{n} \rightarrow S^{n-1} \\
& (v, p) \quad \longmapsto q(v, p)
\end{aligned}
$$

where $q(v, p)=p-\left\langle e_{0}, p\right\rangle A^{-1}(v)$, and $A=\operatorname{Id}_{e_{0}} \times B$, with $B \in \mathrm{SO}(n-1)$, is the unique rotation taking $p$ to a point on the lightlike geodesic $e_{1}+t v, t \in \mathbb{R}$.

Given an embedded submanifold $M$ in $S_{1}^{n}$, the family of projections of $M$ along geodesics refer to the restriction of the families $P_{L}, P_{S}$ and $P_{T}$ to $M$. We still denote this restriction by $P_{L}, P_{S}$ and $P_{T}$ respectively. We have the following result where the term generic is defined in terms of transversality to submanifolds of multi-jet spaces (see for example [3]).

Theorem 3.4 For a residual set of embeddings $\boldsymbol{x}: M \rightarrow S_{1}^{n}$, the families $P_{L}, P_{S}$ and $P_{T}$ are generic families of mappings.

Proof The theorem follows from Montaldi's result in [12] and the fact that $\left.P_{L}\right|_{S_{0}^{n-2} \times M}$, $\left.P_{S}\right|_{S_{1}^{n} \times M-\left\{(v, v) \in S_{1}^{n} \times M\right\}}$ and $\left.P_{T}\right|_{H^{n}(-1) \times M}$ are stable maps. 
Table 1: $\mathcal{A}_{e}$-codimension $\leq 3$ local singularities of map-germs $\mathbb{R}^{2}, 0 \rightarrow \mathbb{R}^{2}, 0$ ([15]).

\begin{tabular}{llc}
\hline Name & Normal form & $\mathcal{A}_{e^{- \text {codimension }}}$ \\
\hline Immersion & $(x, y)$ & 0 \\
Fold & $\left(x, y^{2}\right)$ & 0 \\
Cusp & $\left(x, x y+y^{3}\right)$ & 0 \\
$4_{k}$ & $\left(x, y^{3} \pm x^{k} y\right), k=2,3,4$ & $k-1$ \\
5 & $\left(x, x y+y^{4}\right)$ & 1 \\
6 & $\left(x, x y+y^{5} \pm y^{7}\right)$ & 2 \\
7 & $\left(x, x y+y^{5}\right)$ & 3 \\
$11_{2 k+1}$ & $\left(x, x y^{2}+y^{4}+y^{2 k+1}\right), k=2,3$ & $k$ \\
12 & $\left(x, x y^{2}+y^{5}+y^{6}\right)$ & 3 \\
16 & $\left(x, x^{2} y+y^{4} \pm y^{5}\right)$ & 3 \\
\hline
\end{tabular}

\section{Projections of timelike surfaces in $S_{1}^{3}$}

A projection along a geodesic is singular at $p \in M$ if and only if the geodesic is tangent to $M$ at $p$. Therefore, for spacelike surfaces (whose tangent spaces at all points are spacelike) the projections along timelike and lightlike geodesics are always local diffeomorphisms. The study of projections of spacelike surfaces along spacelike geodesics is similar to that of projections of surfaces in $H^{3}(-1)$ [9]. So we deal here with embedded timelike surfaces $M$ in $S_{1}^{3}$. The projection of $M$ at $p_{0} \in M$ along a given geodesic can be represented locally by a map-germ from the plane to the plane. These map-germs are extensively studied. We refer to [15] for the list of the $\mathcal{A}$-orbits with $\mathcal{A}_{e}$-codimension $\leq 6$, where $\mathcal{A}$ denotes the Mather group of smooth changes of coordinates in the source and target. In Table 1, we reproduce from [15] the list of local singularities of $\mathcal{A}_{e}$-codimension $\leq 3$. Some of these singularities are also called as follows: $4_{2}$ (lips/beaks), $4_{3}$ (goose), 5 (swallowtail), 6 (butterfly), $11_{5}$ (gulls).

We study the local singularities of the projections along the three types of geodesics and characterise them geometrically.

\subsection{Projections along timelike and spacelike geodesics}

It follows from Theorem 3.4 that for generic embeddings of the surface only singularities of $\mathcal{A}_{e}$-codimension $\leq 3$ can occur in the members of the family of orthogonal projections along spacelike and timelike geodesics ( 3 being the dimension of the parameter spaces $S_{1}^{3}$ and $H^{3}(-1)$ respectively). We denote by $P_{S}^{v}$ (resp. $P_{T}^{v}$ ) the map $M \rightarrow H P(v, 0) \cap S_{1}^{3}$ given by $P_{S}^{v}(p)=\pi \circ P_{S}(v, p)\left(\operatorname{resp} . P_{T}^{v}(p)=\pi \circ P_{T}(v, p)\right)$, where $\pi$ is the projection to the second component. The following result follows from Theorem 3.4 .

Proposition 4.1 For a residual set of embeddings $\boldsymbol{x}: M \rightarrow S_{1}^{3}$, the projections $P_{S}^{v}$ 
(resp. $P_{T}^{v}$ ) in the family $P_{S}$ (resp. $P_{T}$ ) have local singularities $\mathcal{A}$-equivalent to one in Table 1. Moreover, these singularities are versally unfolded by the family $P_{S}$ (resp. $\left.P_{T}\right)$.

We seek to derive geometric information on $M$ from the local singularities of the projections. We deal with the members of the family $P_{T}$ and make an observation about those of $P_{S}$.

Given $v \in H^{3}(-1)$ and $p \in S_{1}^{3}$, we denote by $v^{*}$ the parallel transport of $v$ to $p$ along a geodesic orthogonal to $H P(v, 0) \cap S_{1}^{3}$. From the proof of Theorem 3.1 we have $v^{*}=(v-\langle v, p\rangle p) / \sqrt{1+\langle v, p\rangle^{2}}$. We observe that the map $H^{3}(-1) \rightarrow T_{p} S_{1}^{3} \cap H^{3}(-1)$ given by $v \mapsto v^{*} /\left\|v^{*}\right\|$ is a submersion, and the pre-image of a vector $w$ is the curve

$$
C_{w}(t)=\cosh (t) w+\sinh (t) p, t \in \mathbb{R} .
$$

(There is a pencil of hyperplanes defining the same elliptic quadric in $S_{1}^{3}$.) We have the following result where the names of the singularities of $\tilde{P}_{T}^{v}$ are those in Table 1.

Theorem 4.2 Let $M$ be an embedded timelike surface in $S_{1}^{3}$ and $v \in H^{3}(-1)$.

(1) The projection $P_{T}^{v}$ is singular at a point $p \in M$ if and only if $v^{*} \in T_{p} M$.

(2) The singularity of $P_{T}^{v}$ at $p$ is of type cusp or worse if and only if $v^{*}$ is a timelike asymptotic direction at $p$.

(3) The singularities of $P_{T}^{v}$ of Type 5 (i.e., swallowtail) if and only if $v^{*}$ is a timelike asymptotic direction and $p$ is on the flecnodal curve. The singularity of Type 6 if and only if $v^{*}$ is tangent to the flecnodal curve at $p$. At these tangency points, there are generically up to 8 directions on the curve $C_{v^{*}} \subset H^{3}(-1)$ where the singularity becomes of Type 7 .

(4) The singularities of $P_{T}^{v}$ at $p$ is of type $4_{k}, k=2,3,4$, if and only if $p$ is a parabolic point but not a folded singular point of the asymptotic curves and $v^{*}$ is the unique timelike asymptotic direction there. There are up to 12 directions on the curve $C_{v^{*}}$ where the singularity becomes of type $4_{3}$. There are isolated points on the parabolic set where singularity of the projection along these special directions becomes of Type 44 .

(5) At folded singularity of the asymptotic curves there are 12 directions on the curve $C_{v^{*}}$ where the singularity is of Type 16. Away from these directions the singularity is generically of Type $11_{5}$, and for 38 directions on $C_{v^{*}}$ it becomes of Type $11_{7}$. The singularities of Type 12 do not occur in general.

Proof As our study is local in nature, we can make some assumptions about the position of the of surface patch and the choice of the geodesic. We shall assume that the surface patch is at some point $p_{0}$ and that this point is taken by a geodesic $\mathcal{C}_{1}$ to $e_{1}=(0,1,0,0) \in H P\left(e_{0}, 0\right) \cap S_{1}^{3}$. We also suppose that $e_{0}=(1,0,0,0)$ is tangent to $\mathcal{C}_{1}$ at $e_{1}$. The geodesic $\mathcal{C}_{1}$ can then be parametrised by

$$
c_{1}(t)=\cosh (t) e_{1}+\sinh (t) e_{0}, t \in \mathbb{R} .
$$


We take, without loss of generality, the point on the surface to be $p_{0}=(1, \sqrt{2}, 0,0)$. The tangent to $\mathcal{C}_{1}$ at $p_{0}$ is parallel to $w=(\sqrt{2}, 1,0,0)$. Then the vectors $v \in H^{3}(-1)$ satisfying $w=v^{*} /\left\|v^{*}\right\|$ are in the form $v=\left(v_{0}, v_{1}, 0,0\right)$ with $-v_{0}^{2}+v_{1}^{2}=-1$.

The surface patch at $p_{0}$ can be taken in Monge form

$$
\phi(x, y)=\left(1+x, \sqrt{1+(1+x)^{2}-f^{2}(x, y)-y^{2}}, y, f(x, y)\right)
$$

where $f$ is a smooth function in some neighbourhood $U$ of the origin in $\mathbb{R}^{2},(x, y) \in U$ and $f(0,0)=0$. (There is nothing special about the above setting, the results are local in nature and are valid for any $v \in H^{3}(-1)$ and at any point $p_{0} \in M$.)

The projection to the elliptic quadric $H P(v, 0) \cap S_{1}^{3}=H P\left(e_{0}, 0\right) \cap S_{1}^{3}$ in the direction $v$ (with $v$ as above) is then given by

$$
P_{T}^{v}(x, y)=q(v, \phi(x, y))=\frac{1}{\sqrt{1+\langle v, \phi(x, y)\rangle^{2}}}(\phi(x, y)+\langle v, \phi(x, y)\rangle v)
$$

(see Theorem 3.1). As we are interested in the $\mathcal{A}$-singularities of the projection, we can simplify the expression of $P_{T}^{v}$ by projecting further to the tangent space of elliptic quadric $H P\left(e_{0}, 0\right) \cap S_{1}^{3}$ at $e_{1}$. This tangent space is generated by $(0,0,1,0)$ and $(0,0,0,1)$ and we take the projection to this space to be the restriction of the canonical projection $\pi: \mathbb{R}^{4} \rightarrow \mathbb{R}^{2}$, with $\pi\left(x_{0}, x_{1}, x_{2}, x_{3}\right)=\left(x_{2}, x_{3}\right)$. So the modified projection $\tilde{P}_{T}^{e_{0}}=\pi \circ P_{T}^{e_{0}}$ is a map-germ from the plane to the plane given by

$$
\tilde{P}_{T}^{v}(x, y)=\frac{1}{\lambda(x, y, v)}(y, f(x, y)),
$$

with $\lambda(x, y, v)=\left(1+\left(-v_{0}(1+x)+v_{1} \sqrt{1+(1+x)^{2}-f^{2}(x, y)-y^{2}}\right)^{2}\right)^{1 / 2}$. The mapgerm $\tilde{P}_{T}^{v}$ is singular at the origin if and only if $f_{x}(0,0)=0$, if and only if $v^{*}=w \in$ $T_{p_{0}} M$.

We can make successive changes of coordinates in the sources and target and write the appropriate $k$-jet of $\tilde{P}_{T}^{v}$ in the form $(y, g(x, y))$. We can then obtain the conditions on the coefficients of the Taylor expansion of $f$ for $\tilde{P}_{T}^{v}$ to have a given singularity at the origin. The calculations are carried out using Maple. For example the 2 -jet of $\tilde{P}_{T}^{v}$ is $\mathcal{A}$-equivalent to $\left(y, a_{20} x^{2}+a_{21} x y\right)$. We have a fold singularity if and only if $a_{20} \neq 0$. The condition $a_{20}=0$ means that $\phi_{x}(0,0)=\sqrt{2} w$ is an asymptotic direction at $p_{0}$. The other calculations are done similarly but lengthy to reproduce here.

For projections along a spacelike geodesic, we choose the geodesic $\mathcal{C}_{2}$ given by $c_{2}(t)=\cos (t) e_{3}+\sin (t) e_{1}$ and project to the hyperbolic quadric $H P\left(e_{1}, 0\right) \cap S_{1}^{3}$. We take the point $p_{0}=(0, \sqrt{2} / 2,0, \sqrt{2} / 2)$ on the surface (and on $\mathcal{C}_{2}$ ) and project the surface patch around $p_{0}$ along geodesics parallel to $\mathcal{C}_{2}$. We take the surface in Monge form

$$
\phi(x, y)=\left(f(x, y), \sqrt{1+f^{2}(x, y)-\left(\frac{\sqrt{2}}{2}+x\right)^{2}-y^{2}}, y, \frac{\sqrt{2}}{2}+x\right)
$$


with $f(0,0)=f_{x}(0,0)=0$. The modified projection is then a map-germ from the plane to the plane and is given by

$$
\tilde{P}_{S}^{e_{1}}(x, y)=\frac{1}{\sqrt{\left(\frac{\sqrt{2}}{2}+x\right)^{2}+y^{2}-f^{2}(x, y)}}(f(x, y), y) .
$$

We can obtain the conditions for $\tilde{P}_{S}{ }^{e_{1}}$ to have a given singularity at the origin from the coefficients of the Taylor expansion of $f$ and interpret these geometrically. The results are similar to those in Theorem 4.2. One needs to take $v \in S_{1}^{3}$ and replace timelike asymptotic direction by spacelike asymptotic direction in the statement of Theorem 4.2. The numbers of directions in the statements also need changing. In statement (3) we have 4 directions on $C_{v^{*}}$ giving singularities of Type 7 ; in statement (4), there are 2 directions on $C_{v^{*}}$ giving singularities of Type $4_{3}$; in statement (5), there 16 directions on $C_{v^{*}}$ where the singularity becomes of Type $11_{7}$. There are generically no singularities of type 12 or 16.

\subsection{Projections along lightlike geodesics}

We shall give an explicit expression for the projection $P_{L}$ (Definition 3.3) in $S_{1}^{3}$. Consider the sphere $S^{2}=\left\{\left(0, v_{1}, v_{2}, v_{3}\right): v_{1}^{2}+v_{2}^{2}+v_{3}^{2}=1\right\}$ (we shall drop the first coordinate of points in $\left.S^{2}\right)$. Let

$$
T_{\theta}=\left(\begin{array}{ccc}
\cos \theta & -\sin \theta & 0 \\
\sin \theta & \cos \theta & 0 \\
0 & 0 & 1
\end{array}\right), \quad T_{\phi}=\left(\begin{array}{ccc}
\cos \phi & 0 & -\sin \phi \\
0 & 1 & 0 \\
\sin \phi & 0 & \cos \phi
\end{array}\right)
$$

and their composite

$$
T_{(\theta, \phi)}=T_{\theta} \circ T_{\phi}=\left(\begin{array}{ccc}
\cos \theta \cos \phi & -\sin \theta & -\cos \theta \sin \phi \\
\sin \theta \cos \phi & \cos \theta & -\sin \theta \sin \phi \\
\sin \phi & 0 & \cos \phi
\end{array}\right) .
$$

Any point on $S^{2}-(0,0, \pm 1)$ is the image of the point $e_{1}=(1,0,0)$ by a rotation $T(\theta, \phi)$, for some $(\theta, \phi) \in[0,2 \pi] \times]-\frac{\pi}{2}, \frac{\pi}{2}[$. (One can consider other rotations to cover the points $(0,0, \pm 1)$.)

We consider in $S_{1}^{3}$ the rotation $A=\operatorname{Id}_{e_{0}} \times T_{(\theta, \phi)}$. Let $v \in S_{+}^{1}=\left\{\left(1,0, v_{2}, v_{3}\right)\right.$ : $\left.v_{2}^{2}+v_{3}^{2}=1\right\} \subset L C^{*}$. A point $p=\left(p_{0}, p_{1}, p_{2}, p_{3}\right)$ is projected to $q(v, p) \in S^{2}$ along the lightlike geodesic determined by $v$ (see Definition 3.3). The point $q(v, p)$ is the image of $e_{1}$ by a rotation $A$ for some $(\theta, \phi)$. The point $p$ is on the line $q(v, p)+t A(v)$, and we have $p=q(v, p)+p_{0} A(v)$, that is,

$$
\left(\begin{array}{l}
p_{0} \\
p_{1} \\
p_{2} \\
p_{3}
\end{array}\right)=\left(\begin{array}{c}
0 \\
\cos \theta \cos \phi \\
\sin \theta \cos \phi \\
\sin \phi
\end{array}\right)+p_{0}\left(\begin{array}{c}
1 \\
-v_{2} \sin \theta-v_{3} \cos \theta \sin \phi \\
v_{2} \cos \theta-v_{3} \sin \theta \sin \phi \\
v_{3} \cos \phi
\end{array}\right) \text {. }
$$


We suppose that $q(v, p) \neq(0,0, \pm 1)$, this implies that $p_{1}^{2}+p_{2}^{2} \neq 0$. We can then solve the above system for $\theta$ and $\phi$ and get

$$
\begin{aligned}
\cos \phi & =\frac{p_{0} p_{3} v_{3}+\sqrt{1-p_{3}^{2}+p_{0}^{2} v_{3}^{2}}}{1+p_{0}^{2} v_{3}^{2}} & \cos \theta & =\frac{p_{0} p_{2} v_{2}+p_{1} \sqrt{1-p_{3}^{2}+p_{0}^{2} v_{3}^{2}}}{p_{1}^{2}+p_{2}^{2}} \\
\sin \phi & =\frac{p_{3}-p_{0} v_{3} \sqrt{1-p_{3}^{2}+p_{0}^{2} v_{3}^{2}}}{1+p_{0}^{2} v_{3}^{2}} & \sin \theta & =\frac{-p_{0} p_{1} v_{2}+p_{2} \sqrt{1-p_{3}^{2}+p_{0}^{2} v_{3}^{2}}}{p_{1}^{2}+p_{2}^{2}}
\end{aligned}
$$

To analyse the singularities of $P_{L}^{v}$, we take $v=(1,0,0,1)$, the point $p_{0}=e_{1}+v$ so that $q\left(p_{0}, v\right)=e_{1}$. Then a local parametrisation of the surface can be taken in the form

$$
\phi(x, y)=\left(1+x, \sqrt{1+(1+x)^{2}-y^{2}-f^{2}(x, y)}, y, f(x, y)\right)
$$

with $(x, y)$ is in some neighbourhood of the origin in $\mathbb{R}^{2}, f(0,0)=1$ and $f_{x}(0,0)=1$. We consider the modified projection $\tilde{P}_{L}^{v}$ by projecting further to the tangent space of $T_{e_{1}} S^{2}=\left\{\left(0,0, x_{2}, x_{3}\right): x_{2}, x_{3} \in \mathbb{R}\right\}$, which we identify with $\mathbb{R}^{2}$. Then the resulting map-germ from the plane to the plane is given by the last two components of $q(v, p)$, that is $(\sin \theta \cos \phi, \sin \phi)$, with $\sin \theta, \cos \phi, \sin \phi$ as above. That is,

$$
\tilde{P}_{L}^{v}=\left(\frac{y \sqrt{1+(1+x)^{2}-f(x, y)^{2}}\left((1+x) f(x, y)+\sqrt{1+(1+x)^{2}-f(x, y)^{2}}\right.}{\left(1+(1+x)^{2}\right)\left(1+(1+x)^{2}-f^{2}(x, y)\right)}, \frac{f(x, y)-(1+x) \sqrt{1+(1+x)^{2}-f(x, y)^{2}}}{1+(1+x)^{2}}\right) .
$$

We take the 4 -jet of $f$ in the form

$$
j^{4} f(x, y)=1+x+a_{11} y+a_{20} x^{2}+a_{21} x y+a_{22} y^{2}+\sum_{i=0}^{3} a_{3 i} x^{3-i} y^{i}+\sum_{i=0}^{4} a_{4 i} x^{4-i} y^{i} .
$$

The surface patch parametrised by $\phi$ is timelike if and only if $a_{11} \neq 0$. A short calculation shows that the 4 -jet of the projection is $\mathcal{A}$-equivalent to the map-germ

$$
(x, y) \mapsto\left(y, a_{20} x^{2}+a_{21} x y+a_{30} x^{3}+a_{31} x^{2} y-\left(\frac{1}{2} a_{11}^{2}+a_{1} a_{21}-a_{32}\right) x y^{2}+f_{4}(x, y)\right),
$$

with

$f_{4}(x, y)=\left(a_{40}+\frac{1}{2} a_{20}^{2}\right) x^{4}+a_{41} x^{3} y-\left(\frac{1}{2} a_{11}^{2} a_{20}+a_{11}\left(a_{31}+a_{21}\right)+a_{20} a_{22}+\frac{1}{2} a_{21}^{2}-a_{42}\right) x^{2} y^{2}+$ $\left(\frac{1}{2} a_{43}-a_{21} a_{22}-a_{11}\left(a_{22}+a_{32}\right)\right) x y^{3}$.

We have a fold singularity if and only if $a_{20} \neq 0$; a cusp singularity if and only if $a_{20}=0$ and $a_{21} a_{30} \neq 0$; a lips/beaks singularity if and only if $a_{20}=a_{21}=0$ and $a_{30}\left(3\left(a_{1}^{2}+2 a_{1} a_{21}-2 a_{32}\right) a_{30}+2 a_{31}^{2}\right) \neq 0$; a swallowtail singularity if and only if $a_{20}=a_{30}=0$, and $a_{40} \neq 0$.

To interpret these conditions geometrically we look at the $L P L$, given by $\delta(x, y)=0$ in expression (2) in section 2. We calculate the coefficients of the first and second fundamental forms and find that the point $p_{0}$ is on the $L P L$ if and only if

$$
a_{20}\left(a_{20}-2 a_{11} a_{21}+4 a_{20} a_{11}^{4}+4 a_{11}^{2} a_{20}-4 a_{11}^{3} a_{21}+4 a_{11}^{2} a_{22}\right)=0
$$


The asymptotic directions at $p_{0}$ are given by

$$
a_{22} d y^{2}+a_{21} d x d y+a_{20} d x^{2}=0
$$

In particular, the singularity of $P_{L}^{v}$ is worse than fold $\left(a_{20}=0\right)$ if and only if $p_{0} \in L P L$ and $v=\phi_{x}(0,0)$ is a lightlike asymptotic direction. (Then $v$ is also the double principal direction at $p_{0}$, see section 2.) The other asymptotic direction is not lightlike unless the $L P L$ is singular.

When $a_{20}=0$, we assume that $2 a_{11} a_{22}-a_{21}-2 a_{11}^{2} a_{21} \neq 0$, otherwise the point $p_{0}$ is a timelike umbilic point so the $L P L$ is singular there. At such points both asymptotic directions are lightlike and the projection $P_{L}^{v}$ along these directions has a cusp singularity.

Suppose that $a_{20}=0$. Then the 1 -jet of the equation of the $L P L$ is given by

$$
\left(2 a_{11} a_{22}-2 a_{11}^{2} a_{21}-a_{21}\right)\left(-3 a_{11} a_{30} x-\left(-a_{21}^{2}+a_{11} a_{31}\right) y\right) .
$$

As the surface is timelike, $a_{11} \neq 0$. Therefore the singularity of the projection is of type swallowtail at $p_{0}$ or worse (i.e., $a_{30}=0$ ) if and only if the lightlike direction $v$ is tangent to the $L P L$. (This occur at precisely the folded singularities of the configuration of the principal curves [10].)

The point $p_{0}$ is on the parabolic set if and only if $a_{21}^{2}-4 a_{20} a_{22}=0$. So we have a lips/beaks singularity $\left(a_{20}=a_{21}=0\right)$ at the point of tangency of the $L P L$ with the parabolic set. We have thus the following result.

Theorem 4.3 The lightlike projection can have generically the local codimension $\leq 1$ singularities in Table 1. The singularity of $P_{L}^{v}$ at $p_{0} \in M$ is of type

(1) fold if and only if $v$ is not a lightlike asymptotic direction at $p_{0}$;

(2) cusp if and only if $v$ is a lightlike asymptotic direction at $p_{0}$ and is trasverse to the $L P L$;

(3) swallowtail if and only if $v$ is lightlike asymptotic direction at $p_{0}$ and is tangent to the $L P L$;

(4) lips/beaks if and only if $v$ is a lightlike asymptotic direction at $p_{0}$ and $p_{0}$ is the point of tangency of the LPL with the parabolic curve.

\section{Duality}

We prove in this section duality result similar to those in $[8,9]$, and to those in [16] for central projections of surfaces in $\mathbb{R} P^{3}$ and in [2] for orthogonal projections of surfaces in $\mathbb{R}^{3}$.

Let $M$ be an embedded timelike surface in $S_{1}^{3}$. We shall use the duality concepts in $[4,5,6]$, see $\S 6$ for details. We denote by $A_{2}^{\text {par }}$ the ruled surface in $S_{1}^{3}$ swept out by the geodesics in $S_{1}^{3}$ passing through a parabolic point of $M$ and with tangent direction 
there its unique asymptotic direction. We assume that the unique asymptotic direction is not lightlike, so the point is not on the $L P L$.

Let $p(t), t \in I=(-a, a), a>0$, be a local parametrisation of the parabolic set of $M$ and $u(t)$ be the unique unit asymptotic direction at $p(t)$. Recall that $u(t)$ is spacelike on one side of the lightlike point and timelike on the other side of this point ([10] and section 2). The surface $A_{2}^{\text {par }}$ has two connected components given by

$$
\begin{aligned}
& A_{2}^{S-p a r}=\{\cos (s) p(t)+\sin (s) u(t),(s, t) \in I \times J,\langle u(t), u(t)\rangle=1\} \\
& A_{2}^{T-p a r}=\{\cosh (s) p(t)+\sinh (s) u(t),(s, t) \in I \times J,\langle u(t), u(t)\rangle=-1\}
\end{aligned}
$$

with $J=(-b, b)$, for some $b>0$. We also denote by $A_{1} \| A_{1}$ the ruled surface swept out by the spacelike or timelike geodesics in $S_{1}^{3}$ that are tangent to $M$ at two points where the normals to $M$ at such points are parallel. (So the projection $P_{S}$ or $P_{T}$ has a multi-local singularity of type double tangent fold or worse.) The surface $A_{1} \| A_{1}$ has also two component determined by the type of bi-tangent geodesics. We have the following result, whose proof is similar to that of Theorem 3.8 in [9].

Theorem 5.1 Let $M^{*}$ be the $\Delta_{5}$-dual of the timelike surface $M$ embedded in $S_{1}^{3}$.

(1) The $\Delta_{5}$-dual of the surface $A_{2}^{\text {par }}$ is the cuspidaledge of $M^{*}$.

(2) The $\Delta_{5}$-dual of the surface $A_{1} \| A_{1}$ is the self-intersection line of $M^{*}$.

Proof (1) We deal with the $A_{2}^{S-p a r}$ component (the calculations are similar for $A_{2}^{T-p a r}$ and are omitted) and parametrise it as above by $y(s, t)=\cos (s) p(t)+\sin (s) u(t)$. The normal to the surface $A_{2}^{S-p a r}$ is along

$$
y \wedge y_{s} \wedge y_{t}=\cos ^{3}(s) p(t) \wedge u(t) \wedge p^{\prime}(t)+\sin ^{3}(s) p(t) \wedge u(t) \wedge u^{\prime}(t) .
$$

At a generic point $p$ on the parabolic set, the asymptotic direction is transverse to the parabolic set, so $p(t) \wedge u(t) \wedge p^{\prime}(t)$ is along $e(p(t))$. One can prove, following the same arguments in the proof of in Lemma 3.11 in [8], that $p(t) \wedge u(t) \wedge u^{\prime}(t)$ is also along $e(p(t))$. Therefore $y \wedge y_{s} \wedge y_{t}$ is along $e(p(t))$. So the normal to the ruled surface $A_{2}^{S-p a r}$ is constant along the rulings and is given by the normal vector $e(p(t))$ to $M$ at $p(t)$. This means that $A_{2}^{S-p a r}$ is a de Sitter developable surface (i.e, $K \equiv 0$ on $A_{2}^{S-p a r}$ ). Therefore, the $\Delta_{5}$-dual of $A_{2}^{S-p a r}$ is $\{e(p), p$ a parabolic point $\}$. This is precisely the singular set (i.e. the cuspidaledge) of $M^{*}$, the $\Delta_{5}$-dual surface of $M$.

(2) Suppose a multi-local singularity (double tangent fold) occurs at two points $p_{1}$ and $p_{2}$ on $M$. The surface $A_{1} \| A_{1}$ is then a ruled surface generated by spacelike geodesics along a curve $C_{1}$ on $M$ through $p_{1}$, or a curve $C_{2}$ on $M$ through $p_{2}$. The normals to the surface at points on $C_{1}$ and $C_{2}$ that are on the same ruling of $A_{1} \| A_{1}$ are parallel. Let $q(t)$ be a local parametrisation of the curve $C_{1}$ and $u(t)$ be the unit tangent direction to the ruling in $A_{1} \| A_{1}$ through $q(t)$. A parametrisation of $A_{1} \| A_{1}$ is given by

$$
w(s, t)=\cos (s) q(t)+\sin (s) u(t) .
$$


The normal to this surface is along $\cos ^{3}(s) V_{1}(t)+\sin ^{3}(s) V_{2}(t)$ with $V_{1}(t)=q(t) \wedge$ $u(t) \wedge q^{\prime}(t)$ and $V_{2}(t)=q(t) \wedge u(t) \wedge u^{\prime}(t)$. These normals are parallel at two points on any ruling, one point being on the curve $C_{1}$ and the other on $C_{2}$. Therefore $V_{1}(t)$ and $V_{2}(t)$ are parallel, so the normal to the surface $A_{1} \| A_{1}$ is constant along the rulings of this surface. As these are along the normal to the surface at $q(t)$, it follows that the $\Delta_{5}$-dual of $A_{1} \| A_{1}$ is $\left\{e(p), p \in C_{1}\right\}=\left\{e(p), p \in C_{2}\right\}$. This is precisely the self-intersection line of $M^{*}$, the $\Delta_{5}$-dual surface of $M$.

We consider now some components of the bifurcation sets of the families of projections $P_{S}$ and $P_{T}$.

Theorem 5.2 Let $M^{*}$ be the $\Delta_{5}$-dual of a timelike surface $M$ embedded in $S_{1}^{3}$. Then,

(1) The local stratum Bif $\left(P_{S}\right.$, lips/beaks) of the bifurcation set of $P_{S}$, which consists of vectors $v \in S_{1}^{3}$ for which the projection $P_{S}^{v}$ has a lips/beaks singularity, is a ruled surface. The $\Delta_{5}$-dual of Bif $\left(P_{S}\right.$, lips/beaks) is the cuspidaledge of $M^{*}$.

(2) The multi-local stratum Bif $\left(P_{S}, D T F\right)$ of the bifurcation set of $P_{S}$, which consists of vectors $v \in S_{1}^{3}$ for which the projection $P_{S}^{v}$ has a multi-local singularity of type double tangent fold, is a ruled surface. The $\Delta_{5}$-dual of this ruled surface is the self-intersection line of $M^{*}$.

(3) The local stratum Bif $\left(P_{T}\right.$, lips/beaks $)$ of the bifurcation set of $P_{T}$, which consists of vectors $v \in H^{3}(-1)$ for which the projection $P_{T}^{v}$ has a lips/beaks singularity, is a ruled surface. The $\Delta_{1}$-dual of Bif $\left(P_{T}\right.$, lips/beaks $)$ is the cuspidaledge of $M^{*}$.

(4) The multi-local stratum Bif $\left(P_{T}, D T F\right)$ of the bifurcation set of $P_{T}$, which consists of vectors $v \in H^{3}(-1)$ for which the projection $P_{T}^{v}$ has a multi-local singularity of type double tangent fold, is a ruled surface. The $\Delta_{5}$-dual of this ruled surface is the self-intersection line of $M^{*}$.

Proof We prove (1) as the proof of (2) is similar. It follows from Theorem 4.2(5) that the lips/beaks stratum $\operatorname{Bif}\left(P_{S}\right.$, lips/beaks) of the family $P_{S}$ is given by the set of $v \in S_{1}^{3}$ such that $v^{*}$ is an asymptotic direction at a parabolic point $p$, where $v^{*}$ denotes the parallel transport of $v$ to $p$. So $v^{*}=u(t)$ when $v \in B i f\left(P_{S}\right.$, lips/beaks $)$, where $u(t)$ is the unique asymptotic direction at $p(t)$.

We have then

$$
u(t)=v^{*}=\frac{1}{\sqrt{1-\langle v, p(t)\rangle^{2}}}(v-\langle v, p(t)\rangle p(t))
$$

and hence

$$
v=\sqrt{1-\langle v, p(t)\rangle^{2}} u(t)+\langle v, p(t)\rangle p(t) .
$$

If we set $\sin (s)=\langle v, p(t)\rangle$ we get

$$
\operatorname{Bif}\left(P_{P}, \text { lips } / \text { beaks }\right)=\{\cos (s) u(t)+\sin (s) p(t), t \in I, s \in \mathbb{R}\},
$$


which shows that Bif $\left(P_{P}\right.$, lips/beaks $)$ is a ruled surface. For the duality result, following Remark 6.2, we need to find the unit normal vector to Bif $\left(P_{S}\right.$, lips/beaks $)$. Following the same argument in the proof of Theorem 5.1, we find that the normal vector is constant along the rulings of the surface Bif $\left(P_{S}\right.$, lips/beaks $)$ and is along $e(t)$, and the result follows.

Remark 5.3 It is show in [16] that other strata of the bifurcation set of the family of central projections of surface in $\mathbb{R} P^{3}$ are also self-dual. For instance, the set $A_{3}, A_{1}^{3}$ and $A_{1} \times A_{2}$ strata are all self-dual. These results do not hold in our context. If we define the $A_{3}$ set as the surface formed by geodesics through points on the flecnodal curve and with tangent at these points along the associated asymptotic direction, then this surface is not in general a ruled surface. So its dual is not the flecnodal curve on the $\Delta_{5}$-dual surface of $M$. The situation is similar for the other strata.

\section{Appendix}

We require some properties of contact manifolds and Legendrian submanifolds for the duality results in this paper (for more details see for example [1]). Let $N$ be a $(2 n+1)$ dimensional smooth manifold and $K$ be a field of tangent hyperplanes on $N$. Such a field is locally defined by a 1-form $\alpha$. The tangent hyperplane field $K$ is said to be non-degenerate if $\alpha \wedge(d \alpha)^{n} \neq 0$ at any point on $N$. The pair $(N, K)$ is a contact manifold if $K$ is a non-degenerate hyperplane field. In this case $K$ is called a contact structure and $\alpha$ a contact form.

A submanifold $\boldsymbol{i}: L \subset N$ of a contact manifold $(N, K)$ is said to be Legendrian if $\operatorname{dim} L=n$ and $d \boldsymbol{i}_{x}\left(T_{x} L\right) \subset K_{\boldsymbol{i}_{(x)}}$ at any $x \in L$. A smooth fibre bundle $\pi: E \rightarrow M$ is called a Legendrian fibration if its total space $E$ is furnished with a contact structure and the fibres of $\pi$ are Legendrian submanifolds. Let $\pi: E \rightarrow M$ be a Legendrian fibration. For a Legendrian submanifold $\boldsymbol{i}: L \subset E, \pi \circ \boldsymbol{i}: L \rightarrow M$ is called a Legendrian map. The image of the Legendrian map $\pi \circ \boldsymbol{i}$ is called a wavefront set of $\boldsymbol{i}$ and is denoted by $W(\boldsymbol{i})$.

The duality concepts we use in this paper is one of those introduced in $[4,5,6]$, where five Legendrian double fibrations are considered on subsets of the product of two of the pseudo spheres $H^{n}(-1), S_{1}^{n}$ and $L C^{*}$. We recall here only those that are needed in this paper:

(1) (a) $H^{n}(-1) \times S_{1}^{n} \supset \Delta_{1}=\{(v, w) \mid\langle v, w\rangle=0\}$,

(b) $\pi_{11}: \Delta_{1} \rightarrow H^{n}(-1), \quad \pi_{12}: \Delta_{1} \rightarrow S_{1}^{n}$,

(c) $\theta_{11}=\langle d v, w\rangle\left|\Delta_{1}, \theta_{12}=\langle v, d w\rangle\right| \Delta_{1}$.

(5) (a) $S_{1}^{n} \times S_{1}^{n} \supset \Delta_{5}=\{(v, w) \mid\langle v, w\rangle=0\}$,

(b) $\pi_{51}: \Delta_{5} \rightarrow S_{1}^{n}, \pi_{52}: \Delta_{5} \rightarrow S_{1}^{n}$, 
(c) $\theta_{51}=\langle d v, w\rangle\left|\Delta_{5}, \theta_{52}=\langle v, d w\rangle\right| \Delta_{5}$.

Here, $\pi_{i 1}(v, w)=v$ and $\pi_{i 2}(v, w)=w$ for $i=1,5,\langle d v, w\rangle=-w_{0} d v_{0}+\sum_{i=1}^{n} w_{i} d v_{i}$ and $\langle v, d w\rangle=-v_{0} d w_{0}+\sum_{i=1}^{n} v_{i} d w_{i}$. The 1-forms $\theta_{i 1}$ and $\theta_{i 2}, i=1,5$, define the same tangent hyperplane field over $\Delta_{i}$ which is denoted by $K_{i}$.

Theorem $6.1([4,5,6])$ The pairs $\left(\Delta_{i}, K_{i}\right), i=1,5$, are contact manifolds and $\pi_{i 1}$ and $\pi_{i 2}$ are Legendrian fibrations.

Remarks 6.2 (1) Given a Legendrian submanifold $i: L \rightarrow \Delta_{i}, i=1,5$, Theorem 6.1 states that $\pi_{i 1}(\boldsymbol{i}(L))$ is dual to $\pi_{i 2}(\boldsymbol{i}(L))$ and vice-versa. We shall call this duality $\Delta_{i}$-duality.

(2) If $\pi_{11}(\boldsymbol{i}(L))$ is smooth at a point $\pi_{11}(\boldsymbol{i}(u))$, then $\pi_{12}(\boldsymbol{i}(u))$ is the normal vector to the hypersurface $\pi_{11}(\boldsymbol{i}(L)) \subset H_{+}^{n}(-1)$ at $\pi_{11}(\boldsymbol{i}(u))$. Conversely, if $\pi_{12}(\boldsymbol{i}(L))$ is smooth at a point $\pi_{12}(\boldsymbol{i}(u))$, then $\pi_{11}(\boldsymbol{i}(u))$ is the normal vector to the hypersurface $\pi_{12}(\boldsymbol{i}(L)) \subset S_{1}^{n}$. The same properties hold for the $\Delta_{5}$-duality.

Acknowledgement. The results in this paper were established during the visit of FT to Hokkaido University in October 2007. The visit was supported by a Royal Society International Outgoing Short Visit grant 2007/R2. We would like to thank the Royal Society for their support.

\section{References}

[1] V. I. Arnol'd, S. M. Gusein-Zade and A. N. Varchenko, Singularities of Differentiable Maps vol. I. Birkhäuser, 1986.

[2] J. W. Bruce and M. C. Romero-Fuster, Duality and orthogonal projections of curves and surfaces in Euclidean 3-space. Quart. J. Math. Oxford 42 (1991), 433-441.

[3] M. Golubitsky and V. Guillemin, Stable mappings and their singularities. Graduate Texts in Mathematics, Vol. 14. Springer-Verlag, New York-Heidelberg, 1973.

[4] S. Izumiya, Timelike hypersurfaces in de Sitter space and Legendrian singularities. $J$. Math. Sciences. 144 (2007), 3789-3803.

[5] S. Izumiya, Legendrian dualities and spacelike hypersurfaces in the lightcone. To appear in Moscow Mathematical Journal.

[6] S. Izumiya and M. Takahashi, Spacelike parallels and evolutes in Minkowski pseudospheres. J. Geometry and Physics 57 (2007), 1569-1600.

[7] S. Izumiya, M. Takahashi and F. Tari, Folding maps on spacelike and timelike surfaces and duality. Preprint, 2007. Available from http://maths.dur.ac.uk/ dma0ft/Publications.html, or http://eprints3.math.sci.hokudai.ac.jp/view/type/preprint.html 
[8] S. Izumiya and F. Tari, Projections of surfaces in the hyperbolic space to hyperhorospheres and hyperplanes. Rev. Mat. Iberoamericana 24 (2008), 895-920.

[9] S. Izumiya and F. Tari, Projections of surfaces in the hyperbolic space along horocycles. Preprint, 2008. Available from http://maths.dur.ac.uk/ dma0ft/Publications.html, or http://eprints3.math.sci.hokudai.ac.jp/view/type/preprint.html

[10] S. Izumiya and F. Tari, Pairs of foliations on timelike surfaces in the de Sitter space. Preprint, 2008. Available from http://maths.dur.ac.uk/ dma0ft/Publications.html, or http://eprints3.math.sci.hokudai.ac.jp/view/type/preprint.html

[11] B. Karliğa and M. Savas, Orthogonal projections to a $k$-plane in hyperbolic, de Sitter and spherical $n$-spaces. Preprint, 2007.

[12] J. A. Montaldi, On generic composites of maps. Bull. London Math. Soc. 23 (1991), $81-85$.

[13] B. O'Neill, Semi-Riemannian geometry. With applications to relativity. Pure and Applied Mathematics, 103. Academic Press, Inc., New York, 1983.

[14] J. G. Ratcliffe, Foundations of hyperbolic manifolds. Graduate Texts in Mathematics, 149. Springer-Verlag, New York, 1994.

[15] J. H. Rieger, Families of maps from the plane to the plane. J. London Math. Soc. 36 (1987), 351-369.

[16] O. P. Shcherbak, Projectively dual space curves and Legendre singularities. Sel. Math. Sov 5 (1986), 391-421.

S. Izumiya, Department of Mathematics, Hokkaido University, Sapporo 060-0810, Japan

e-mail: izumiya@math.sci.hokudai.ac.jp

F. Tari, Department of Mathematical Sciences, Durham University, Science Laboratories, South Road, Durham DH1 3LE, UK

e-mail: farid.tari@durham.ac.uk 